\title{
Multi-view gait recognition on curved trajectories
}

\author{
D. López-Fernández, F.J. Madrid-Cuevas, A. Carmona-Poyato, R. Muñoz-Salinas and R. Medina-Carnicer
}

\begin{abstract}
Appearance changes due to viewing angle changes cause difficulties for most of the gait recognition methods. In this paper, we propose a new approach for multi-view recognition, which allows to recognize people walking on curved paths. The recognition is based on 3D angular analysis of the movement of the walking human. A coarse-to-fine gait signature represents local variations on the angular measurements along time. A Support Vector Machine is used for classifying, and a sliding temporal window for majority vote policy is used to smooth and reinforce the classification results. The proposed approach has been experimentally validated on the publicly available "Kyushu University 4D Gait Database". The results show that this new approach achieves promising results in the problem of gait recognition on curved paths.
\end{abstract}

Keywords-Gait recognition, $3 D$ descriptor, independent-view, curved paths.

\section{INTRODUCTION}

Researches on human gait as a biometric feature for identification have received a lot of attention due to the advantage that it can operate from a distance and can be applied discreetly without needing the active participation of the observed individual [1]. However, gait recognition performance is significantly affected by changes in various covariate conditions such as clothing [2], camera viewpoint [3], [4], load carrying [5], and walking speed [6].

According to camera viewpoint, the previous work can be categorized into two approaches: view-dependent and viewindependent approaches. View-dependent approaches assume that the viewpoint will not change while walking [7]-[12]. In such methods, a change in the appearance, caused by a viewpoint change, adversely affects to the recognition [13]. For example, when a subject walks along a curved trajectory, the observation angle between the walking direction of the subject and the camera optical axis is gradually changed at all frames during a gait cycle. Fig. 1 shows this problem and the influence of a curved path on the silhouette appearance.

This paper presents a new approach for multi-view gait recognition which allows to identify people walking along both curved and straight paths. Some potential applications of this work is smart video surveillance (e.g. bank offices, government facilities, or underground stations) and access control or monitoring in special or restricted areas (e.g. military bases or medical isolation zones where subjects wear special clothing that does not allow to show the face or use the fingerprint).

The rest of the paper is structured as follows. After presenting in Section II the related work, we describe our proposed framework for gait recognition in Section III Section IV] is devoted to the experimental results. And, finally, we conclude this paper in Section $\mathrm{V}$

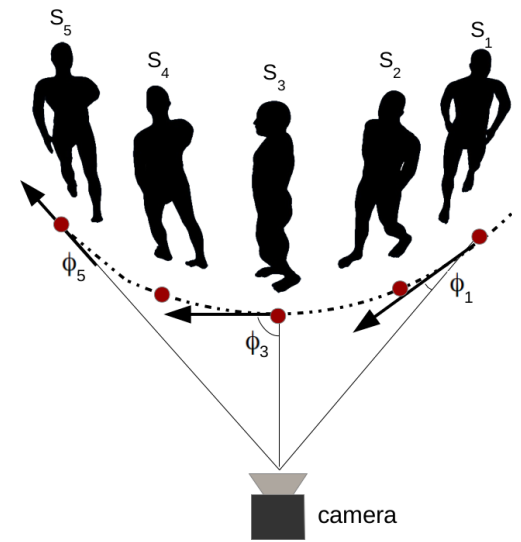

Figure 1. In a curved path, the observation angle between the walking direction of the subject and optical axis of the camera is gradually changed, which affects the silhouette appearance.

\section{RELATED WORK}

Appearance changes due to viewing angle changes cause difficulties for most of the gait recognition methods. This situation cannot be easily avoided in practical applications. There are three major approach categories to sort out this problem, namely: (1) approaches that construct 3D gait information through multiple calibrated cameras; (2) approaches that extract gait features which are invariant to viewing angle changes; (3) approaches whose performance relies on learning mapping/projection relationship of gaits under various viewing angles [3].

Approaches of the first category are represented by [4], [14], [15]. In [14], a 3D approximation of a Visual Hull (VH) [16] is used to design a multi-modal recognition approach. Although a VH model is computed, a gait recognition scheme based on silhouette analysis is applied, which restricts a large amount of discriminant information because the recognition is based on single view silhouette analysis, instead of analyse 3D information.

Another approach that applies image-based rendering on a $3 \mathrm{D} \mathrm{VH}$ model to reconstruct gait features under a required viewing angle is presented in [15]. This approach tries to classify the motion of a human in a view-independent way, but it also has two drawbacks. On the one hand it considers only straight paths to estimate the position and orientation of a virtual camera. Tests were performed only on straight path motions. On the other hand, not all the 3D information available in the $\mathrm{VH}$ is used, because feature images are extracted from 2D images rendered only from a single view.

In [4], an observation angle at each frame of a gait sequence is estimated from the walking direction, by fitting a 2D polynomial curve to the foot points. Virtual images are synthesized from a $3 \mathrm{D}$ model, so that the observation 
angle of a synthesized image is the same that the observation angle for the real image of the subject, which is identified by using affine moment invariants extracted from images as gait features. The advantage of this method is that the setup assumes multiple cameras for training, but only one camera for testing. However, as in the above two works, despite 3D models are used, descriptors are computed from silhouettes and they based on 2D information, so that 3D information is discarded. On the other hand, shadows on the floor complicate the estimation of the foot points in silhouette images. This approach requires to split the sequence into gait cycles and assumes that the gait phase of the first frame of a gait cycle of a subject is the same for each person in the database.

Approaches of the second category extract gait features which are invariant to viewing angle change. In [17], it is described a method to generate a canonical view of gait from any arbitrary view. This method can work with a single calibrated camera but the synthesis of a canonical view is only feasible from a limited number of initial views. The performance is significantly dropped when the angle between image plane and sagittal plane is large.

In [18], a method based on homography to compute viewnormalized trajectories of body parts obtained from monocular video sequences was proposed. But this method efficiently works only for a limited range of views. Planar homography has also been used to reduce the dependency between the motion direction and the camera optical axis [19], however this method seems not to be applicable when the person is walking nearly parallel to the optical axis. In [20] viewinvariant features are extracted from GEI. Only parts of gait sequences that overlap between views are selected for gait matching, but this approach cannot cope with large view angle changes under which gait sequences of different views can have little overlap.

A self-calibrating view-independent gait recognition based on model-based gait features is proposed in [21]. The poses of the lower limbs are estimated based on markerless motion estimation. Then, they are reconstructed in the sagittal plane using viewpoint rectification. This method has two main drawbacks that are worth mentioning: 1) the estimation of the poses of the limbs is not robust from markerless motion; 2) it is not applicable for frontal view because the poses of the limbs become untraceable; and 3) this method assume that subjects walk along a straight line segment.

The approaches of the third category rely on learning mapping/projection relationship of gaits under various viewing angles. The trained relationship may normalize gait features from different viewing angles into shared feature spaces. An example from this category can be read in [22], where LDAsubspaces are learned to extract discriminative information from gait features under each viewing angle.

A View Transformation Model (VTM) was introduced by [23] to transform gait features from different views into the same view. The method of Makihara et al. [23] creates a VTM based on frequency-domain gait features, obtained through Fourier Transformation. To improve the performance of this method, Kusakunniran et al. A sparse-regression-based VTM for gait recognition under various views is also proposed in [3]. However, this method cannot deal with changes in the direction of motion.

Although methods of the third category have better ability to cope with large view angle changes compared to other works, some common challenges are the following [3]: (1) performance of gait recognition decreases as the viewing angle increases; (2) since the methods rely on supervised learning, it will be difficult for recognizing gait under untrained/unknown viewing angles, (3) these methods implicitly assume that people walk along straight paths and that their walking direction does not change during a single gait cycle (i.e., that people do not walk along curved trajectories).

\section{PROPOSED FRAMEWORK}

This work presents a method to recognize walking humans independently of the viewpoint and regardless direction changes. Our approach aims to extract 3D dynamical information of gait. The body human region is vertically divided into 3D stacked areas of the same size called slices and then we compute the centroid of each slice. The gait feature is composed by a set of acute angles between the line joining each pair of consecutive centroids and the $\mathrm{z}$-axis $(\mathrm{z}-$ axis extends up) in R3.

The proposed algorithm consist of four steps that predict the identity of a walking human a time $t$. Following are described these steps in detail.

- Tracking of the walking human.

- Descriptor generation.

- $\quad$ Signature update.

- Classification.

\section{A. Tracking}

We assume a set of $N$ calibrated cameras. Since cameras have been calibrated, the internal and external camera parameters are known. We also assume the floor to be flat and its position in $3 \mathrm{D}$ space to be known.

The first step of our algorithm is to determine the location of the individual in the scene. For that, we start by obtaining the principal axis of the silhouette for each camera view $i$ by Principal Component Analysis. Next, for each view, we back-project this line in order to get the plane $\pi_{i} \in$ $\left\{\pi_{0}, \pi_{1}, \ldots, \pi_{N-1}\right\}$, as Figure 2 shows.

It is assumed a function $f: \mathbb{R}^{3} \mapsto \mathbb{R}^{3}$ to map from camera local coordinates to scene world coordinates. Then we map each plane $\pi_{i}$ from local camera coordinates to scene world coordinates. Let us denote $r_{i, j}$ as the intersection line between the planes $\pi_{i}$ and $\pi_{j}$, where $0 \leqslant i<N$ and $0 \leqslant j<N$.

We denote $F$ as a set of candidate foot points, obtained by intersecting the lines $r_{i, j}$ with the floor plane, without repetition, so that the cardinality of the set is $|F|=\left(\begin{array}{c}N \\ 2\end{array}\right)$. Finally, the location of the individual is denoted by:

$$
P=\frac{1}{|F|} \sum_{i=0}^{|F|} F_{i} .
$$




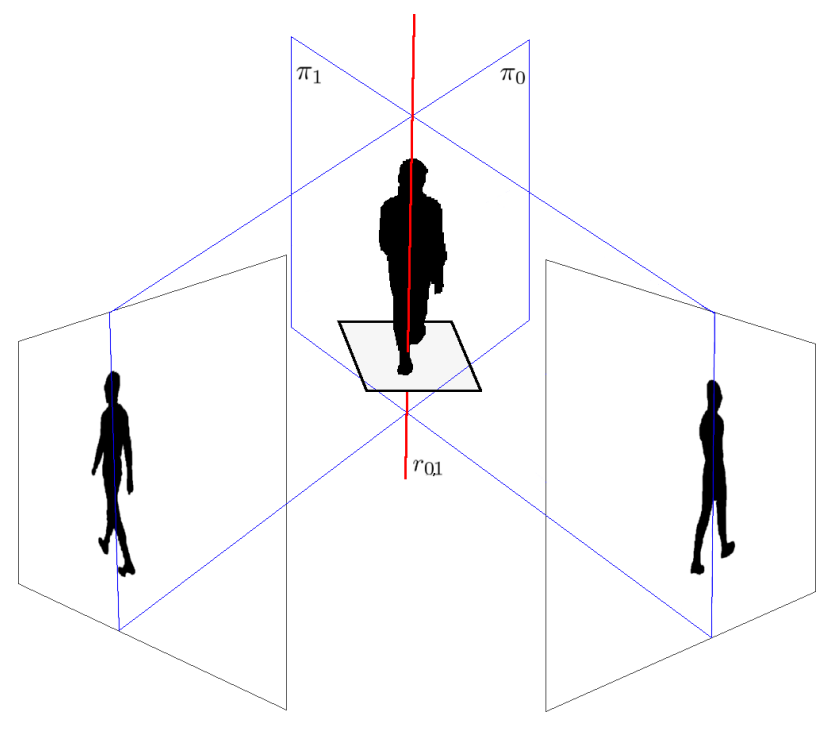

Figure 2. The principal axis of the silhouettes is back-projected to get a plane. Then, the location of the individual in the scene is determined by the intersection between the line of intersection of the two planes and the floor plane.

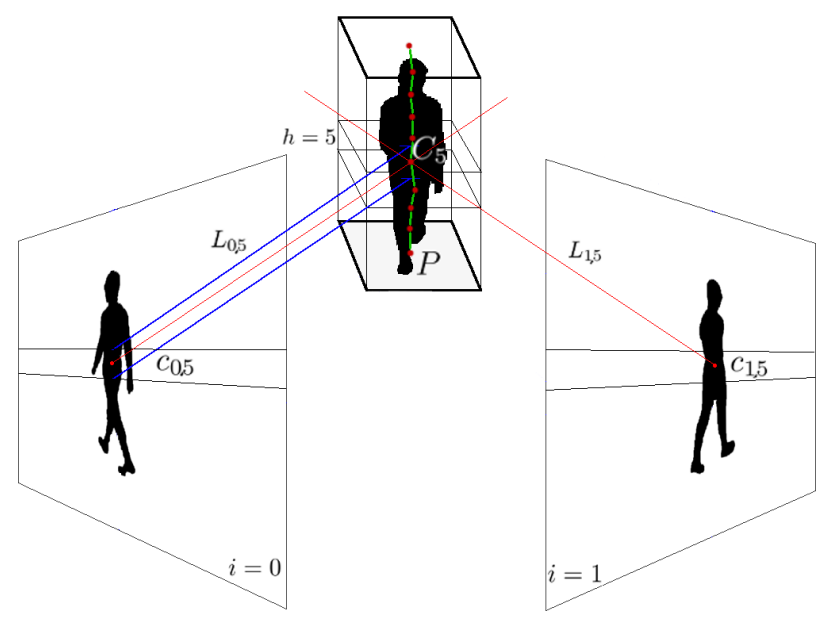

Figure 3. The centroid $C_{h}$ is obtained by finding the point closest to the set of rays $\left\{L_{i, h} \mid 0 \leqslant i<N\right\}$. See main text for further details.

\section{B. Descriptor generation}

Given a foot point $P:\left(P_{x}, P_{y}, 0\right)$, the $3 \mathrm{D}$ scene is vertically divided into $H \in \mathbb{N}^{+}$parts, called slices. Let us denote $p^{i, h}$ and $p^{i, h+1}$ as the projections on the image view $i$ of the 3D points $\left(P_{x}, P_{y}, h \frac{Z}{H}\right)$ and $\left(P_{x}, P_{y},(h+1) \frac{Z}{H}\right)$ respectively, where $Z$ is the total height of the 3D scene. We compute the $2 \mathrm{D}$ centroid $c_{i, h}=\{\bar{x}, \bar{y}\}$ on the bounding box enclosing the pixels $\left(0, p_{y}^{i, h+1}\right)$ and $\left(w, p_{y}^{i, h}\right)$, where $w$ is the width of the image.

Then, using the $2 \mathrm{D}$ centroid $c_{i, h}$ and the calibration data for the view $i$, we can backproject the ray $L_{i, h}$ passing through the image view point $c_{i, h}$. In order to obtain an approximation of the 3D centroid $C_{h}$ of the slice $h$ in the scene, we find the point closest to the set of rays $\left\{L_{i, h} \mid 0 \leqslant i<N\right\}$. We propose to solve it by minimizing the sum of squared distances.

Next we define the angle between the normal vector to the floor plane $(\vec{Z}=(0,0,1))$ and the vector joining each pair of consecutive centroids as:

$$
\begin{gathered}
\alpha_{h}=\arccos \left(\frac{\vec{Z} \cdot \overrightarrow{C_{h} C_{h+1}}}{\overline{C_{h} C_{h+1}}}\right), 0 \leqslant h<H-2, \\
\beta_{h}=\min \left\{\alpha_{h}, 180-\alpha_{h}\right\},
\end{gathered}
$$

where $\overrightarrow{C_{h} C_{h+1}}$ is the vector connecting the $C_{h}$ and $C_{h+1}$ centroids. Thus, for each instant $t$, our descriptor is a tuple of angular measurements that we can define as:

$$
D_{H, t}=\left(\beta_{(0, t)}, \beta_{(1, t)}, \ldots, \beta_{(H-2, t)}\right) .
$$

If the slice $h$ is empty (e.g. slices above the head) or $\left|\left\{L_{i, h}\right\}\right|<2, C_{h}$ cannot be estimated. In such cases, to preserve the height of the subject as feature, we set $\beta_{h, t}=0$.

\section{Signature update}

The first step of our classification system is the generation of the gait descriptor $D_{(H, t)}$ at time $t$. Then, the gait signature can be built as a time series of gait descriptors obtained from the $3 \mathrm{D}$ reconstructed gait sequence.

In order to combine different description levels, we propose a coarse-to-fine refinement. We define the number of levels as:

$$
0<l \leqslant\left\lfloor\log _{2} H\right\rfloor,
$$

so that the first level descriptor contains features extracted from the scene divided into 2 slices, the second level descriptor contains features extracted from the scene divided into $H=2^{2}$ slices, and so on until we have divided the scene into $H=2^{l}$ slices. We can now concatenate the level descriptors to represent our coarse-to-fine descriptor as:

$$
\mathscr{D}_{(l, t)}=\left(D_{(2, t)}, D_{\left(2^{2}, t\right)}, \ldots, D_{\left(2^{l}, t\right)}\right) .
$$

The gait signature is a temporal pattern of gait, a sample that feeds a classifier producing a class label corresponding to the identity of a particular person. Our signature is updated at every moment of the walking, and it allows to take place a synchronous classifying process. Thus, we define the gait signature $\mathscr{G}$ on a sliding temporal window of size $L$. Let us denote $\mathscr{G}$ as:

$$
\mathscr{G}_{(l, t)}=\left(\mathscr{D}_{(l, t-L+1)}, \ldots, \mathscr{D}_{(l, t-1)}, \mathscr{D}_{(l, t)}\right),
$$

which consists of a concatenation of $L$ consecutive descriptors. In other words, our gait signature is updated at each instant of the gait by concatenating successive gait descriptors into a sliding temporal window of size $L$.

Our gait signature has several advantages that are worth mentioning. First, the gait phase of the first frame of a gait sequence of a subject does not have to be the same for each person in the database. Second, it does not require the sequence to be split into gait cycles, and therefore it is not necessary to 
estimate the gait period. This makes our method less restrictive compared to other techniques from the literature such as [3], [4] [24] among others.

\section{Classification}

The gait signature $\mathscr{G}_{(l, t)}$ is in fact the feature vector used for classification. Each feature vector is assigned to a class label that corresponds to one of the person in the database.

We adopt the subspace Component and Discriminant Analysis, based on Principal Component Analysis (PCA) and Linear Discriminant Analysis (LDA), which seeks to project the original features to a subspace of lower dimensionality so that the best data representation and class separability can be achieved simultaneously [25]. Then we use a Support Vector Machine (SVM) [26] for training and classification.

The gait signature is based on the $L$ previous volumes, and a possibly different class label can be produced for each new gait signature at each time. In order to smooth and reinforce the results over time, we use a majority vote policy over a sliding temporal window of size $W$. Our recognition algorithm provides the identity of the person as soon as possible. However, as the gait signature information is computed on $L$ previous volumes, the use of this window causes a delay of $L+(W-1)$ frames in obtaining the identity. The majority voting system over a sliding temporal window is represented in Fig. 4.

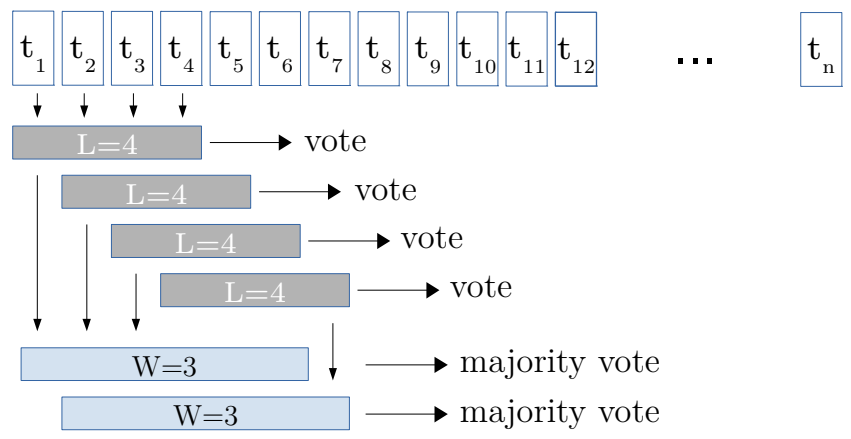

Figure 4. Majority vote policy over a sliding temporal window. In the example, the size of the signature is set to $\mathrm{L}=4$, and the size of the voting window is set to $\mathrm{W}=3$.

\section{OVERVIEW OF THE EXPERIMENTS}

In order to validate our approach, we carry out diverse experiments on the publicly available "Kyushu University 4D Gait Database" [4]. With these experiments we try to answer, among others, the following questions:

- Is our descriptor a valid approach to recognize walking humans independently of the viewpoint, even with curved trajectories?

- What level of refinement for our coarse-to-fine gait descriptor is required to achieve the best recognition rate?

- What is the influence of the sliding temporal window for majority voting policy on the recognition rate?

- How many cameras are needed to achieve good performance?

\section{A. Dataset description}

"Kyushu University 4D Gait Database" (KY4D) 1] [4], it is composed of sequential 3D models and image sequences of 42 subjects walking along four straight and two curved trajectories. The sequences were recorded by 16 cameras, at a resolution of $1032 \times 776$ pixels. The studio setup is shown in Figure 5. Despite 3D models are available, we do not use them, because this work relies on camera calibration to obtaining $3 \mathrm{D}$ information.

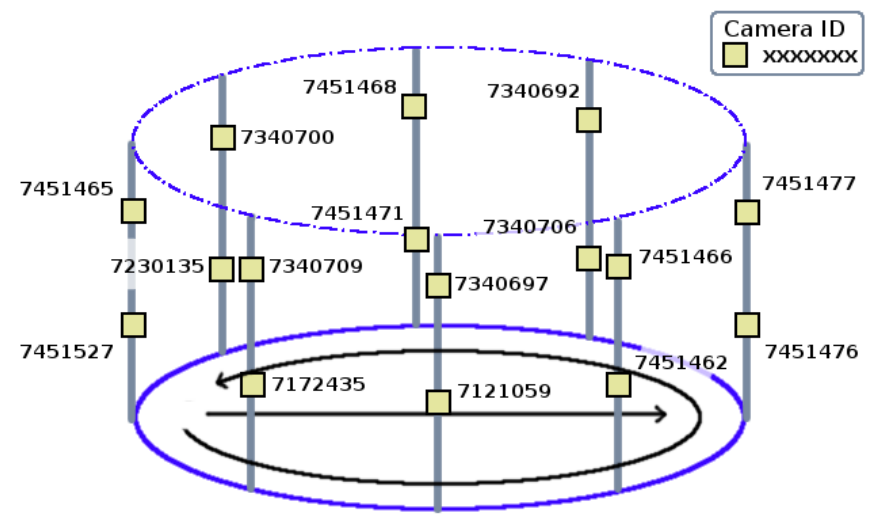

Figure 5. Experimental setup of KY4D. This image is part of the public release of KY4D Gait Database.

As can be seen in Figure 5, KY4D gait sequences are captured by 16 cameras forming rings at two heights. The lower level comprises the cameras $\{7451527,7172435,7121059$, $7451462,7451476,7340706,7451471,7230135\}$, whereas the upper level comprises the cameras $\{7451465,7340709$, 7340697, 7451466, 7451477, 7340692, 7451468, 7340700\}.

\section{B. Experimental results}

Next we need to determine the value of several parameters of our method. According to the length of the gait signature, we set to $L=20$ the number of frames where our descriptor is computed, because this value roughly matches with the average length of a gait cycle. Regarding the number of levels (see Section III-C, we tested $l$ from 1 ( 2 slices) to $6\left(2^{6}\right.$ slices $)$.

We use a leave-one-out cross-validation strategy. Thus, each fold is composed by 42 sequences (one sequence per actor) for testing and by the remaining five sequences of each actor (i.e. $42 \times 5$ sequences) for training. To make the choice of SVM parameters independent of the sequence test data, we cross-validate the SVM parameters on the training set. Note that curved paths are sometimes longer than straight paths. Moreover, some subjects walk faster than others and therefore cause a greater number of votes. To cope with this issue, we normalize by class the results of each trajectory.

In order to achieve the best data representation and class separability simultaneously, we apply PCA+LDA to the training and test data (see Section III-D). With regard to PCA, we only retain $95 \%$ of the variance. In the classification step,

\footnotetext{
${ }^{1}$ Publicly available at: http://robotics.ait.kyushu-u.ac.jp/researche.php?content $=\mathrm{db}$
} 


\begin{tabular}{|r|r|r|}
\hline$l$ & PCA & PCA+LDA \\
\hline \hline 1 & 5.99 & N.A \\
\hline 2 & 31.54 & 32.92 \\
\hline 3 & 56.98 & 56.38 \\
\hline 4 & 74.75 & 74.68 \\
\hline 5 & 80.21 & $\mathbf{8 1 . 4 0}$ \\
\hline 6 & 79.56 & 80.69 \\
\hline
\end{tabular}

Table I. CORRECT CLASSIFICATION RATE [\%] ON THE LOWER SET OF CAMERAS FOR SEVERAL VALUES OF THE PARAMETER $l$. THE SIZE OF THE SLIDING TEMPORAL WINDOW FOR MAJORITY VOTING IS SET TO $W=1$. BEST RESULT IS MARKED IN BOLD. (SEE MAIN TEXT FOR FURTHER DETAILS.)

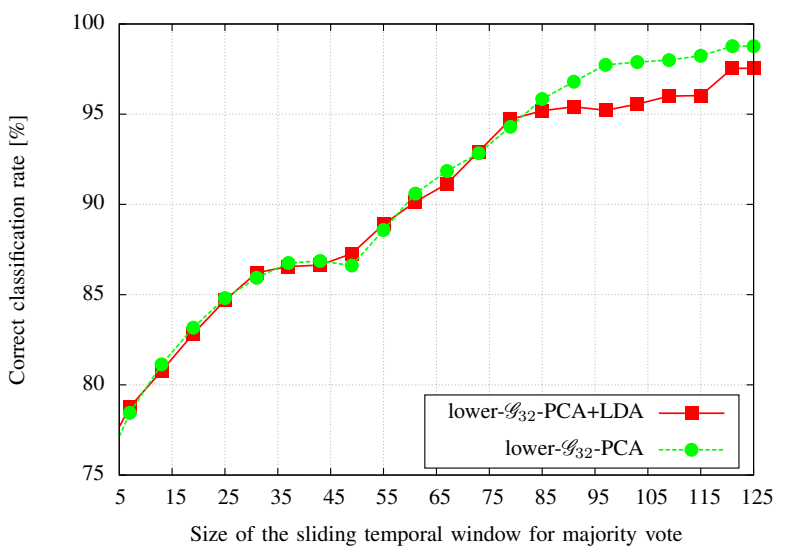

Figure 6. Performance of our descriptor on the lower level of cameras of KY4D database for different lengths of the majority voting window.

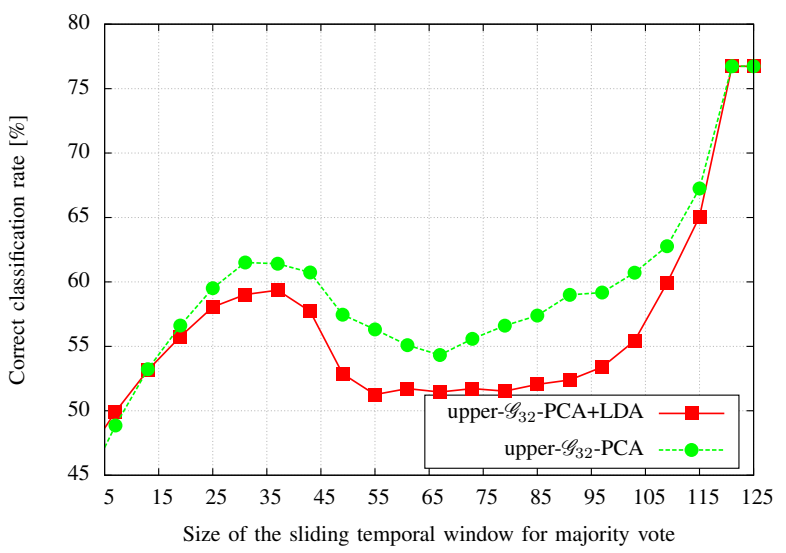

Figure 7. Performance of our descriptor on the upper level of cameras of KY4D database for different lengths of the majority voting window.

we tested several SVM kernels, and finally we selected a CSVC SVM with Radial Basis Function since we obtained better results than with linear, polynomial, or sigmoid kernels.

The recognition rate on the lower set of cameras for several values of the parameter $l$ is shown in Table II It also shows the effect of the dimensionality reduction on the recognition rate. In this experiment, for the sake of simplicity, we disabled the sliding temporal window for majority voting $(W=1)$. We obtained the best results with $l=5$ and PCA+LDA. It must be note that besides the recognition rate, the number of features is considerably lower with PCA+LDA than with PCA.

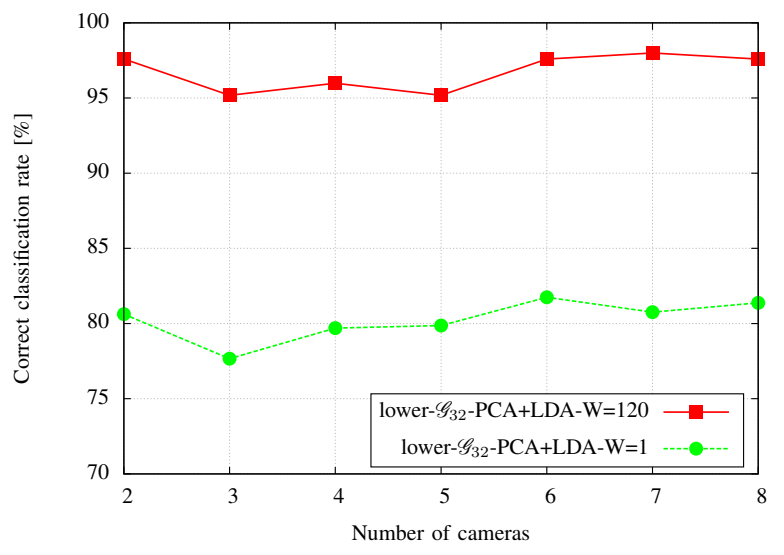

Figure 8. Performance of our descriptor for an increasing number of cameras.

We next conducted experiments in which we applied the sliding temporal window for majority voting policy. As can be seen in Figure 6 and Figure 7, the use of a majority voting policy over a sliding temporal window significantly improves the performance of our method. However, the performance obtained with the lower set of cameras is greater than with the upper set (see Section IV-A). The size of the window is limited by the number of available gait signatures for each sequence.

The results of Table $\amalg$ shows detailed results for the leaveone-out experiment, with $l=5$. We show the effect of applying the sliding temporal window for majority voting compared with $W=1$ (disabled window). As can be seen, we have obtained better results with the lower set of cameras than with the upper set. This could be due to the tilt of the cameras. This issue is left for a future study.

In order to determine the number of cameras that should be employed and its effect on the performance, we have designed a leave-one-out cross validation experiment. We selected the signature configuration that achieved the best performance in the previous experiments and then we tested it with a variable number of cameras of the lower set in the range 2 to 8 . As can be seen in Figure 8, with just 2 calibrated cameras, our method is able to correctly classify up to $95 \%$ of individuals, independently of the path, even with curved trajectories.

\section{CONCLUSION}

This paper has proposed a new approach to recognize walking humans independently of the viewpoint. Our approach allow people to walk freely in the scene, in contrast to others view-independent approaches which restrict the view change to a few angles.

A new rotation invariant gait descriptor has been proposed to cope with rotation changes on curved trajectories, while preserving enough discriminatory information from the gait. Our descriptor focuses on capturing 3D dynamical information of gait.

This approach does not require the sequence to be split into gait cycles, and the results are smoothed and reinforced over time by using a sliding temporal window for majority voting 


\begin{tabular}{|c|c|c|c|c|c|c|c|}
\hline & \multicolumn{4}{|c|}{ Straight paths } & \multicolumn{2}{|c|}{ Curved paths } & \\
\hline Experiment & t1 & $\mathrm{t} 2$ & $\mathrm{t} 3$ & $\mathrm{t} 4$ & t5 & t6 & AVG \\
\hline upper- $\mathscr{G}_{32}-\mathrm{PCA}-\mathrm{W}=1$ & 44.63 & 51.80 & 46.78 & 48.13 & 28.74 & 58.45 & 46.42 \\
\hline upper- $\mathscr{G}_{32}$-PCA-W=120 & 70.73 & 78.04 & 82.92 & 87.80 & 54.34 & 85.00 & 76.47 \\
\hline upper- $\mathscr{G}_{32}-\mathrm{PCA}+\mathrm{LDA}-\mathrm{W}=1$ & 49.85 & 57.07 & 51.12 & 52.68 & 29.70 & 56.05 & 49,41 \\
\hline upper- $\mathscr{G}_{32}-\mathrm{PCA}+\mathrm{LDA}-\mathrm{W}=120$ & 80.48 & 80.48 & 90.24 & 82.92 & 52.17 & 72.50 & 76,46 \\
\hline lower- $\mathscr{G}_{32}-\mathrm{PCA}-\mathrm{W}=1$ & 84.86 & 87.73 & 88.97 & 89.49 & 52.24 & 78.02 & 80.21 \\
\hline lower- $\mathscr{G}_{32}-\mathrm{PCA}-\mathrm{W}=120$ & 95.12 & 100 & 100 & 100 & 97.82 & 100 & 98.82 \\
\hline lower- $\mathscr{G}_{32}-\mathrm{PCA}+\mathrm{LDA}-\mathrm{W}=1$ & 88.24 & 89.82 & 89.10 & 90.30 & 52.42 & 78.52 & 81.40 \\
\hline lower- $\mathscr{G}_{32}-\mathrm{PCA}+\mathrm{LDA}-\mathrm{W}=120$ & 97.56 & 100 & 100 & 100 & 91.30 & 97.50 & 97.72 \\
\hline
\end{tabular}

Table II. CORRECT CLASSIFICATION RATE ON KY4D [\%]. EACH COLUMN CORRESPONDS TO A TEST TRAJECTORY, USING THE REMAINING TRAJECTORIES AS TRAINING SET. EACH ROW CORRESPONDS TO A DIFFERENT CONFIGURATION OF THE GAIT DESCRIPTOR. EACH ENTRY CONTAINS THE PERCENTAGE OF CORRECT RECOGNITION FOR EACH TUPLE TRAJECTORY-SETUP.

policy. Experimental results show that our method is able to reach a correct classification rate up to $95 \%$.

\section{ACKNOWLEDGMENT}

This work has been developed with the support of the Research Projects called TIN2012-32952 and BROCA both financed by Science and Technology Ministry of Spain and FEDER.

\section{REFERENCES}

[1] W. Hu, T. Tan, L. Wang, and S. Maybank, "A survey on visual surveillance of object motion and behaviors," IEEE Transactions on Systems, Man, and Cybernetics, Part C: Applications and Reviews, vol. 34, no. 3, pp. 334-352, Aug 2004.

[2] M. A. Hossain, Y. Makihara, J. Wang, and Y. Yagi, "Clothing-invariant gait identification using part-based clothing categorization and adaptive weight control," Pattern Recognition, vol. 43, no. 6, pp. 2281 - 2291, 2010

[3] W. Kusakunniran, Q. Wu, J. Zhang, and H. Li, "Gait recognition under various viewing angles based on correlated motion regression," IEEE Transactions on Circuits and Systems for Video Technology, vol. 22, no. 6, pp. 966-980, June 2012.

[4] Y. Iwashita, K. Ogawara, and R. Kurazume, "Identification of people walking along curved trajectories," Pattern Recognition Letters, vol. 48, no. 0 , pp. $60-69,2014$, celebrating the life and work of Maria Petrou.

[5] S. Singh and K. Biswas, "Biometric gait recognition with carrying and clothing variants," in Pattern Recognition and Machine Intelligence, ser. Lecture Notes in Computer Science. Springer Berlin Heidelberg, 2009, vol. 5909, pp. 446-451.

[6] A. Tsuji, Y. Makihara, and Y. Yagi, "Silhouette transformation based on walking speed for gait identification," in IEEE Conference on Computer Vision and Pattern Recognition (CVPR), June 2010, pp. 717-722.

[7] A. Kale, N. Cuntoor, B. Yegnanarayana, A. Rajagopalan, and R. Chellappa, "Gait analysis for human identification," in Audio- and VideoBased Biometric Person Authentication. Springer Berlin Heidelberg, 2003, vol. 2688, pp. 706-714.

[8] M.-H. Cheng, M.-F. Ho, and C.-L. Huang, "Gait analysis for human identification through manifold learning and hmm," Pattern Recognition, vol. 41, no. 8, pp. 2541-2553, Aug. 2008.

[9] C. P. Lee, A. W. Tan, and S. C. Tan, "Gait recognition via optimally interpolated deformable contours," Pattern Recognition Letters, vol. 34, no. 6 , pp. $663-669,2013$.

[10] S. D. Choudhury and T. Tjahjadi, "Gait recognition based on shape and motion analysis of silhouette contours," Computer Vision and Image Understanding, vol. 117, no. 12, pp. 1770 - 1785, 2013.

[11] M. Hofmann, S. Bachmann, and G. Rigoll, " $2.5 \mathrm{~d}$ gait biometrics using the depth gradient histogram energy image," in IEEE Fifth International Conference on Biometrics: Theory, Applications and Systems (BTAS), Sept 2012, pp. 399-403.
[12] S. Sivapalan, D. Chen, S. Denman, S. Sridharan, and C. Fookes, "Gait energy volumes and frontal gait recognition using depth images," in International Joint Conference on Biometrics (IJCB). Washington DC, USA: IEEE, Oct 2011, pp. 1-6.

[13] S. Yu, D. Tan, and T. Tan, "Modelling the effect of view angle variation on appearance-based gait recognition," in Computer Vision - ACCV 2006, ser. Lecture Notes in Computer Science. Springer Berlin Heidelberg, 2006, vol. 3851, pp. 807-816.

[14] G. Shakhnarovich, L. Lee, and T. Darrell, "Integrated face and gait recognition from multiple views," in IEEE Computer Society Conference on Computer Vision and Pattern Recognition (CVPR), vol. 1, 2001, pp. I-439-I-446 vol.1.

[15] R. Bodor, A. Drenner, D. Fehr, O. Masoud, and N. Papanikolopoulos, "View-independent human motion classification using image-based reconstruction," Image and Vision Computing, vol. 27, no. 8, pp. 11941206, 2009.

[16] A. Laurentini, "The visual hull concept for silhouette-based image understanding," IEEE Transactions on Pattern Analysis and Machine Intelligence, vol. 16, no. 2, pp. 150-162, Feb 1994.

[17] A. Kale, A. Roy-Chowdhury, and R. Chellappa, "Towards a view invariant gait recognition algorithm," in IEEE Conference on Advanced Video and Signal Based Surveillance, July 2003, pp. 143-150.

[18] F. Jean, A. B. Albu, and R. Bergevin, "Towards view-invariant gait modeling: Computing view-normalized body part trajectories," Pattern Recognition, vol. 42, no. 11, pp. 2936 - 2949, 2009.

[19] S. Jeong, T.-h. Kim, and J. Cho, "Gait recognition using description of shape synthesized by planar homography," The Journal of Supercomputing, vol. 65 , no. 1, pp. 122-135, 2013.

[20] J. Han, B. Bhanu, and A. Roy-Chowdhury, "A study on view-insensitive gait recognition," in IEEE International Conference on Image Processing (ICIP), vol. 3, Sept 2005, pp. III-297-300.

[21] M. Goffredo, I. Bouchrika, J. Carter, and M. Nixon, "Self-calibrating view-invariant gait biometrics," IEEE Transactions on Systems, Man, and Cybernetics, Part B: Cybernetics, vol. 40, no. 4, pp. 997-1008, Aug 2010.

[22] N. Liu and Y.-P. Tan, "View invariant gait recognition," in IEEE International Conference on Acoustics Speech and Signal Processing (ICASSP), March 2010, pp. 1410-1413.

[23] Y. Makihara, R. Sagawa, Y. Mukaigawa, T. Echigo, and Y. Yagi, "Gait recognition using a view transformation model in the frequency domain," in Computer Vision - ECCV 2006, ser. Lecture Notes in Computer Science. Springer Berlin Heidelberg, Jan. 2006, no. 3953, pp. 151-163.

[24] K. Bashir, T. Xiang, and S. Gong, "Cross-view gait recognition using correlation strength," in Proceedings of the British Machine Vision Conference. BMVA Press, 2010, pp. 109.1-109.11.

[25] P. Huang, C. Harris, and M. Nixon, "Recognising humans by gait via parametric canonical space," Artificial Intelligence in Engineering, vol. 13, no. 4, pp. 359 - 366, 1999.

[26] C. J. Burges, "A tutorial on support vector machines for pattern recognition," Data Mining and Knowledge Discovery, vol. 2, no. 2, pp. 121-167, 1998. 